\title{
一种菱形结构的二维塊合振荡器阵列及其 在有源天线阵中的应用
}

\author{
翟龙军"，姜永华，刘隆和 \\ 海军航空工程学院电子信息工程系, 烟台 264001 \\ *E-mail: zhailongjun@163.com \\ 收稿日期: 2007-04-09; 接受日期: 2007-06-19 \\ 武器装备预研基金(批准号: 51307060103)资助项目
}

\begin{abstract}
摘要传统的二维互注入锁相耦合振荡器阵列采用矩形栅格结构, 可以实 现无移相器的波束形成和扫描. 矩形结构阵列相邻阵元相位差限制在 $\left[-90^{\circ}\right.$, $90^{\circ}$ ] 内, 当阵元间距为半波长时, 其水平和俯仰波束扫描范围限制在偏离法 线方向 $30^{\circ}$ 以内. 论文提出了一种菱形结构的二维耦合振荡器阵列, 给出了 其相位控制方法，并对相位控制误差进行了分析. 在对菱形结构的耦合振荡 器阵列的稳定性进行分析后, 给出了稳定条件, 对阵列的稳定过程和相位控 制方法进行了计算机仿真，对理论分析结果进行了验证。该菱形结构耦合振 荡器阵列, 在水平和俯仰方向上相邻阵元的相位差可达 $\left[-180^{\circ}, 180^{\circ}\right]$, 具有 比矩形栅格结构更宽的波束扫描范围.
\end{abstract}

关键词

耦合振荡器阵列

菱形结构

有源天线阵

稳定性

相位控制

1993 年, Liao和York ${ }^{[1]}$ 提出了一种用于有源天线阵的一维互注入锁相耦合振荡器阵列. 这 种耦合振荡器阵列不使用移相器, 而是通过反对称调整阵列两端的振荡器的自由振荡频率控 制阵面的相位分布, 实现波束扫描. 这种阵列的动力学行为通过一组耦合的非线性微分方程 来描述 ${ }^{[2]}$, 这些方程可以通过描述注频锁相现象的Adler方程 ${ }^{[3]}$ 推导得到. Liao和York利用贴片 天线之间的耦合, 制作了 4 元耦合振荡器阵列 ${ }^{[1]}$. Pogorzelski等人 ${ }^{[4]}$ 通过振荡器谐振电路之间 的耦合, 制作实现了 7 元耦合振荡器阵列. 这些实验结果验证了这种方案的可行性. 随后, 这 种不用移相器而利用耦合振荡器实现相位分布控制的方法被推广到矩形栅格结构的二维阵列, 如图 1(a)所示, 得到了类似的结果 ${ }^{[5,6]}$. 理论和实验表明, 对于矩形栅格结构的二维阵列, 可以 通过控制位于边界上的振荡器的自由振荡频率实现阵面的相位分布控制 ${ }^{[7,8]}$.

基于耦合振荡器阵列的有源天线阵曾一度被认为类似于通过慢波线实现相位控制的频率 扫描天线. 事实上，频率扫描阵列依靠馈入信号的频率变化改变阵内相位差，当波束指向不同 
时, 辐射到空间的信号频率是变化的. 耦合振荡器阵列依靠调整振荡器自由振荡频率改变阵 内相位差, 只要阵列的平均振荡频率不变, 当波束指向不同时, 辐射到空间的信号频率是不变 的. 频率扫描阵列不能实现同一指向的波束内信号的频率捷变. 耦合振荡器阵列从原理上讲 是可以的. 只要改变阵列平均振荡频率的同时, 保持自由振荡频率相对关系不变, 那么波束指 向不会发生变化, 而辐射到空间中的信号的频率可以是变化的, 从而可以实现同一个波束指 向内的频率捷变.

常规的矩形栅格结构二维耦合振荡器阵列波束扫描范围较窄, 为了增加扫描范围, 通常 采用对耦合振荡器的输出信号进行倍频的方法 ${ }^{[9]}$, 这样又增加了系统复杂度. Pogorzelski提出 了六角形和三角形结构, 如图 1(b)和 1(c)所示. 三角形结构具有比矩形栅格结构更短的稳定时 间, 而六角形结构的稳定时间比矩形栅格结构慢, 而且口径效率低, 三角形结构和六角形结构 要实现比矩形栅格结构更宽的波束扫描范围, 也必须对振荡器的输出信号进行倍频 $[10]$. 为了 扩展二维耦合振荡器阵列的波束扫描范围, 本文提出了一种菱形结构的二维耦合振荡器阵列, 给出了阵列的相位控制方法, 并对稳定性进行了分析. 利用菱形结构的二维耦合振荡器阵列 实现的有源天线阵, 在水平和俯仰方向上相邻阵元的相位差可达 $\left[-180^{\circ}, 180^{\circ}\right]$, 具有比矩形栅 格结构更宽的波束扫描范围.

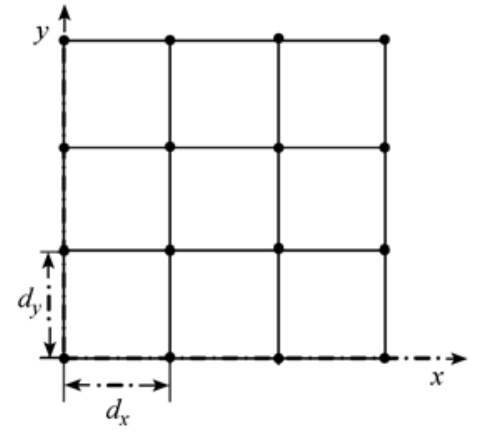

(a)

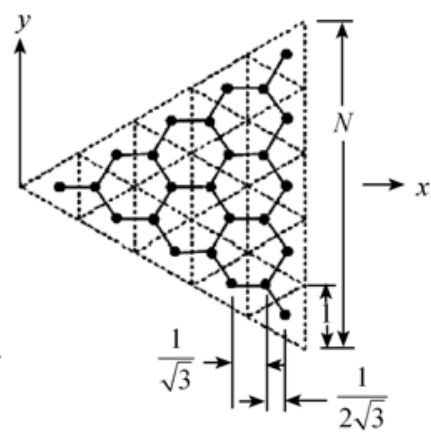

(b)

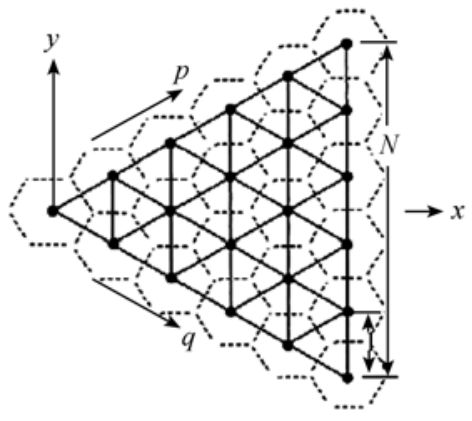

(c)

图 1 二维耦合振荡器阵列的拓扑结构

（a）矩形栅格结构; (b) 六角形结构; (c) 三角形结构

\section{1 非线性振荡器互注入锁相原理}

非线性振荡器之间的互注入锁相原理如图 2 所示. 两个von der Pol非线性振荡器通过耦合 网络实现互相耦合, 如图 2(a)所示. 描述振荡器相位和幅度的微分方程如(1)式所示 ${ }^{[2]}$.

$$
\begin{gathered}
\left\{\begin{array}{l}
\dot{A}_{1}=\mu \Delta \omega_{m}\left(p_{1}^{2}-A_{1}^{2}\right) A_{1}+\Delta \omega_{m} A_{2} \cos \left(\theta_{2}-\theta_{1}+\Phi\right), \\
\dot{\theta}_{1}=\omega_{1}+\Delta \omega_{m} \frac{A_{2}}{A_{1}} \sin \left(\theta_{2}-\theta_{1}+\Phi\right),
\end{array}\right. \\
\left\{\begin{array}{l}
\dot{A}_{2}=\mu \Delta \omega_{m}\left(p_{2}^{2}-A_{2}^{2}\right) A_{2}+\Delta \omega_{m} A_{1} \cos \left(\theta_{1}-\theta_{2}+\Phi\right), \\
\dot{\theta}_{2}=\omega_{2}+\Delta \omega_{m} \frac{A_{1}}{A_{2}} \sin \left(\theta_{1}-\theta_{2}+\Phi\right),
\end{array}\right.
\end{gathered}
$$




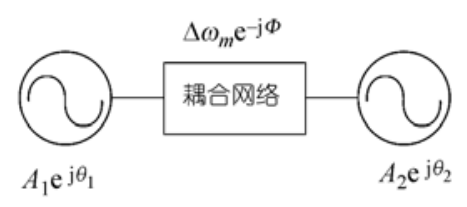

(a)

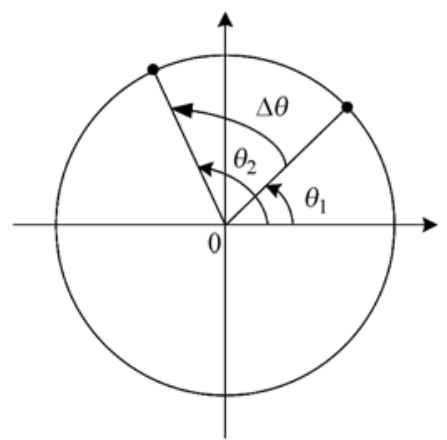

(b)

图 2 非线性振荡器互注入锁相原理

其中, $A_{1}, \theta_{1}$ 和 $A_{2}, \theta_{2}$ 分别表示两个振荡器的振荡幅度和相位, $\Phi$ 表示耦合网络的相移. $\omega_{i}, p_{i}(i=1,2)$ 为两个振荡器的自由振荡频率和自由振荡幅度. $\Delta \omega_{m}$ 表示锁相带宽, 与耦合网 络的耦合强度、振荡器品质因数和自由振荡频率有关. $\mu$ 为描述振荡器非线性的参数.

当耦合强度 $k \ll 1$ 时, 根据幅度动力学方程, 耦合时振荡器的幅度接近于自由振荡幅度 $\left(A_{i} \approx p_{i}, i=1,2\right)$, 振荡器的状态变化主要受相位动力学方程的影响. 当两个振荡器的自由振 荡频率和自由振荡幅度相等时 $\left(p_{1}=p_{2}\right)$, 描述振荡器状态变化的方程可以简化为

$$
\left\{\begin{array}{l}
\dot{\theta}_{1}=\omega_{1}+\Delta \omega_{m} \sin \left(\theta_{2}-\theta_{1}+\Phi\right), \\
\dot{\theta}_{2}=\omega_{2}+\Delta \omega_{m} \sin \left(\theta_{1}-\theta_{2}+\Phi\right) .
\end{array}\right.
$$

令 $\Delta \theta=\theta_{2}-\theta_{1}, \Delta \omega=\omega_{2}-\omega_{1}$, 则有

$$
\Delta \dot{\theta}=\Delta \omega-2 \Delta \omega_{m} \sin (\Delta \theta) \cos \Phi .
$$

如图 2(b)所示, 当 $k \cos \Phi>0$ 并且 $|\Delta \omega| \leqslant 2 \Delta \omega_{m} \cos \Phi$ 时, (3)式具有 $\Delta \dot{\theta}=0$ 的稳定状态, 即两个 振荡器同步锁相. 锁相相位差如(4)式所示, 形成的相位差范围为 $\left[-90^{\circ}, 90^{\circ}\right]$.

$$
\Delta \theta=\sin ^{-1}\left(\frac{\Delta \omega}{2 \Delta \omega_{m} \cos \Phi}\right) .
$$

从(4)式可知, $\Delta \theta$ 主要取决于 $\Delta \omega$, 调整 $\Delta \omega$, 可以调整锁相相位差的大小.

根据非线性振荡器互注入锁相原理, York, Pogorzelski和Heath等人 ${ }^{[5,6]}$ 将其推广到二维阵 列, 提出了如图 1(a)所示的矩形栅格结构, 这也是目前二维耦合振荡器阵列的常规结构. 矩形 栅格结构的耦合振荡器阵列的相位动力学方程为

$$
\dot{\theta}_{i j}=\omega_{i j}+\Delta \omega_{m} \sum_{\substack{m=i \pm 1 \\ n=j \pm 1}} \sin \left(\theta_{m n}-\theta_{i j}+\Phi\right), i=1, \cdots, M, j=1, \cdots, N .
$$

其中, $\theta_{i j}, \quad \omega_{i j}$ 分别为第 $i j$ 个振荡器的相位和自由振荡频率; $\theta_{m n}$ 为与第 $i j$ 个振荡器相邻的振 荡器的相位. 阵列边界条件为

$$
\theta_{0 j}=\theta_{1 j}-\Phi, \theta_{M+1, j}=\theta_{M j}-\Phi, \theta_{i 0}=\theta_{i 1}-\Phi, \theta_{i, N+1}=\theta_{i, N}-\Phi .
$$


矩形栅格结构的二维耦合振荡器阵列形成的平面相位分布具有如下的形式:

$$
\theta_{m n}=\omega_{\mathrm{ref}} t+(m-1) \theta_{g x}+(n-1) \theta_{g y} .
$$

在耦合相位为 0 时, 阵列的稳定性条件为 ${ }^{[7]}$

$$
\left|\theta_{g x}\right| \leqslant \pi / 2 \text {, 且 }\left|\theta_{g y}\right| \leqslant \pi / 2,
$$

即相邻阵元的相位差范围限制在 $\left[-90^{\circ}, 90^{\circ}\right]$ 内. 当阵元间距为半波长时, 波束扫描范围限制在 $\left[-30^{\circ}, 30^{\circ}\right]$ 之间. 实际在工程实现时, 由于噪声和自由振荡频率误差的影响, 相邻阵元之间的 相位差往往达不到 $90^{\circ}$, 即波束扫描范围达不到 $\left[-30^{\circ}, 30^{\circ}\right]$. 为了增加扫描范围, 通常采用对耦 合振荡器的输出信号进行倍频的方法 ${ }^{[9]}$, 这样又增加了系统复杂度.

\section{2 菱形结构二维耦合振荡器阵列}

矩形栅格结构的二维耦合振荡器阵列中在行和列方向上相邻的振荡器之间都有耦合网络 连接, 这就决定了其行方向和列方向上相邻阵元的相位差不会超过 $\pm 90^{\circ}$, 从而阵元间距为半 波长时其波束扫描范围不会超过 $\pm 30^{\circ}$. 基于柾格型结构的上述缺点, 为了扩展阵列的波束扫 描范围, 我们提出了菱形结构. 菱形结构阵列在行和列方向上相邻的阵元并不直接发生耦合, 而是通过中间阵元发生相互作用，这使得行和列方向上的相邻阵元在稳定状态时的可形成的 相位差比矩形栅格结构要大，从而具有更宽的波束扫描范围.

\section{1 相位动力学方程}

菱形结构的二维耦合振荡器阵列如图 3 所示. 为方便起见, 我们将振荡器用黑点表示, 耦 合网络用振荡器之间的连接线表示，并假定所有的耦合网络都具有相同的参数.

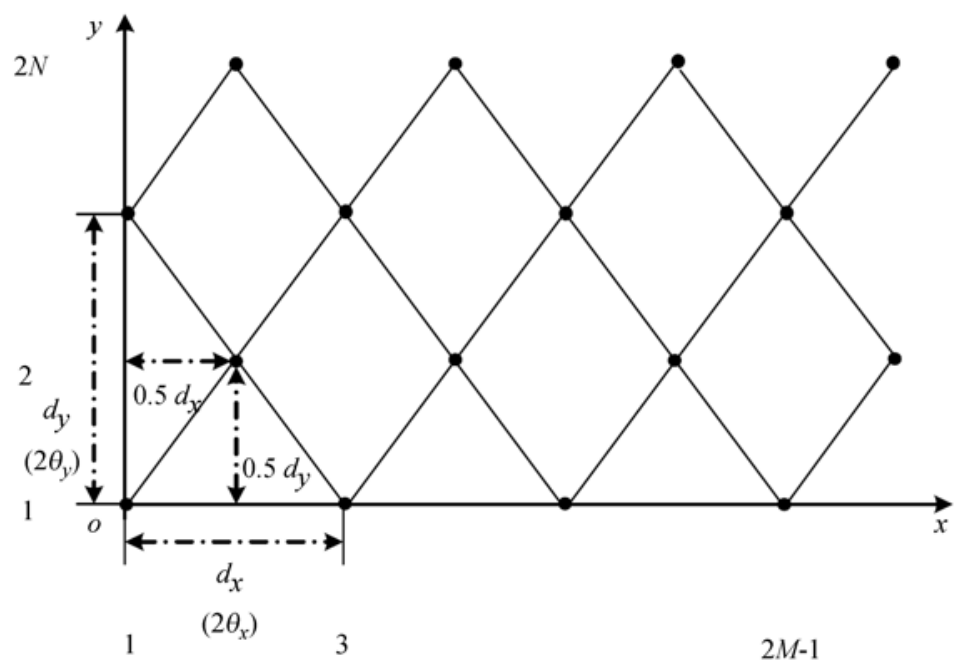

图 3 菱形结构二维耦合振荡器阵列

根据互注入锁相原理, 可以得到菱形结构的二维耦合振荡器阵列的相位动力学方程为 


$$
\left\{\begin{array}{c}
\dot{\theta}_{2 m-1,2 n-1}=\omega_{2 m+1,2 n+1}+\Delta \omega_{m} \sin \left(\theta_{2 m, 2 n}-\theta_{2 m-1,2 n-1}+\Phi\right)+\Delta \omega_{m} \sin \left(\theta_{2 m-2,2 n-2}-\theta_{2 m-1,2 n-1}+\Phi\right) \\
+\Delta \omega_{m} \sin \left(\theta_{2 m-2,2 n}-\theta_{2 m-1,2 n-1}+\Phi\right)+\Delta \omega_{m} \sin \left(\theta_{2 m, 2 n-2}-\theta_{2 m-1,2 n-1}+\Phi\right), \\
\dot{\theta}_{2 m, 2 n}=\omega_{2 m, 2 n}+\Delta \omega_{m} \sin \left(\theta_{2 m+1,2 n+1}-\theta_{2 m, 2 n}\right)+\Delta \omega_{m} \sin \left(\theta_{2 m-1,2 n-1}-\theta_{2 m, 2 n}\right) \\
+\Delta \omega_{m} \sin \left(\theta_{2 m-1,2 n+1}-\theta_{2 m, 2 n}\right)+\Delta \omega_{m} \sin \left(\theta_{2 m+1,2 n-1}-\theta_{2 m, 2 n}\right),
\end{array}\right.
$$

其中, $m=1,2, \cdots, M ; n=1,2, \cdots, N ; \quad \theta_{m n}$ 和 $\omega_{m n}$ 为位于 $(m, n)$ 点的振荡器的相位和自由振荡频 率; $\Delta \omega_{m}$ 为锁相带宽; $\Phi$ 为耦合网络的相移. 阵列的边界条件为

$$
\left\{\begin{array}{l}
\theta_{00}=\theta_{01}=\theta_{10}=\theta_{11}-\Phi, \\
\theta_{2 M+1,2 N+1}=\theta_{2 M+1,2 N-1}=\theta_{2 M-1,2 N+1}=\theta_{2 M, 2 N}-\Phi, \\
\theta_{2 m, 0}=\theta_{2 m-2,0}=\theta_{2 m-1,1}-\Phi, \quad m=2,3, \cdots, M, \\
\theta_{2 m+1,2 N+1}=\theta_{2 m-1,2 N+1}=\theta_{2 m, 2 N}-\Phi, \quad m=1,2, \cdots, M-1, \\
\theta_{0,2 n}=\theta_{0,2 n-2}=\theta_{1,2 n-1}-\Phi, \quad n=2,3, \cdots, N, \\
\theta_{2 M+1,2 n+1}=\theta_{2 M+1,2 n-1}=\theta_{2 M, 2 n}-\Phi, \quad n=1,2, \cdots, N-1 .
\end{array}\right.
$$

图 3 中的二维耦合振荡器阵列在适当控制阵元的自由振荡频率时, 所有阵元可以达到稳 定的同步振荡状态. 图 4 是 $\omega_{m n}=2.45 \mathrm{GHz}$, 锁相带宽为 $160 \mathrm{MHz}, \Phi=0$, 振荡器初始相位随 机取值时, $4 \times 4$ 阵列稳定过程的仿真结果. 从图中可以看出, 经过约 $20 \mathrm{~ns}$ 的瞬态过程, 阵列达 到同步.

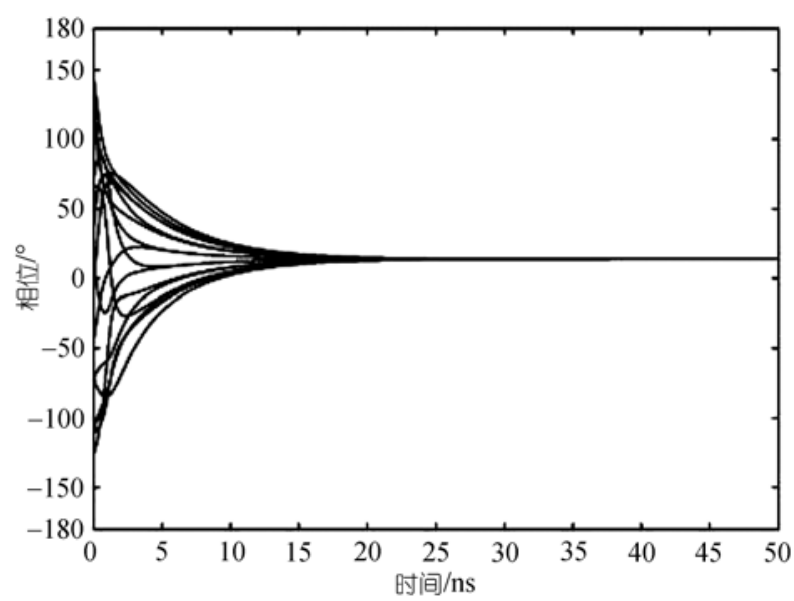

图 $44 \times 4$ 阵列的稳定过程

当阵列同步时, 有 $\dot{\theta}_{m n}=\omega_{\mathrm{syn}}$. 将(7)式中的微分方程依次相加得到

$$
\sum \dot{\theta}_{m n}=\sum \omega_{m n}
$$

当阵列达到同步时, 所有振荡器的振荡频率相同, 因此有

$$
\omega_{\mathrm{sys}}=\frac{1}{2 N M} \sum \omega_{m n} .
$$


说明阵列达到同步时，同步振荡频率为所有振荡器自由振荡频率的平均值.

\section{2 相位控制方法}

对于阵元按照图 3 排列的有源天线阵, 要形成平面相位分布, 则阵元的相位应该具有如下 的形式:

$$
\left\{\begin{array}{l}
\theta_{2 m-1,2 n-1}=\omega_{\mathrm{ref}} t+(2 m-2) \theta_{x}+(2 n-2) \theta_{y}, \\
\theta_{2 m, 2 n}=\omega_{\mathrm{ref}} t+(2 m-1) \theta_{x}+(2 n-1) \theta_{y},
\end{array}\right.
$$

其中, $\omega_{\mathrm{ref}}$ 为参考频率; $\theta_{x}, \theta_{y}$ 为常数. 要使得(11)式成为(7)式的稳态解, 将(11)式代入到(7)式 中, 可以得到频率控制参量应该满足的条件为

$$
\left\{\begin{aligned}
\omega_{2 m-1,2 n-1}=\omega_{\text {ref }}-\Delta \omega_{m} \sin \left(\theta_{\Sigma}+\Phi\right)-\Delta \omega_{m} \sin \left(-\theta_{\Sigma}+\Phi\right) & \\
& \quad-\Delta \omega_{m} \sin \left(-\theta_{\Delta}+\Phi\right)-\Delta \omega_{m} \sin \left(\theta_{\Delta}+\Phi\right), \\
\omega_{2 m, 2 n}= & \omega_{\text {ref }}-\Delta \omega_{m} \sin \left(\theta_{\Sigma}+\Phi\right)-\Delta \omega_{m} \sin \left(-\theta_{\Sigma}+\Phi\right) \\
& \quad-\Delta \omega_{m} \sin \left(-\theta_{\Delta}+\Phi\right)-\Delta \omega_{m} \sin \left(\theta_{\Delta}+\Phi\right), \\
\omega_{1,1}=\omega_{\text {ref }}-\Delta \omega_{m} \sin \left(\theta_{\Sigma}+\Phi\right), & \\
\omega_{2 M, 2 N}= & \omega_{\text {ref }}-\Delta \omega_{m} \sin \left(-\theta_{\Sigma}+\Phi\right), \\
\omega_{2 m-1,1}= & \omega_{\text {ref }}-\Delta \omega_{m} \sin \left(\theta_{\Sigma}+\Phi\right)-\Delta \omega_{m} \sin \left(-\theta_{\Delta}+\Phi\right), \quad m=2,3, \cdots, M, \\
\omega_{2 m, 2 N}= & \omega_{\text {ref }}-\Delta \omega_{m} \sin \left(-\theta_{\Sigma}+\Phi\right)-\Delta \omega_{m} \sin \left(\theta_{\Delta}+\Phi\right), \quad m=1,2, \cdots, M-1, \\
\omega_{1,2 n-1}= & \omega_{\text {ref }}-\Delta \omega_{m} \sin \left(\theta_{\Sigma}+\Phi\right)-\Delta \omega_{m} \sin \left(\theta_{\Delta}+\Phi\right), \quad n=2,3, \cdots, N, \\
\omega_{2 M, 2 n}= & \omega_{\text {ref }}-\Delta \omega_{m} \sin \left(-\theta_{\Sigma}+\Phi\right)-\Delta \omega_{m} \sin \left(-\theta_{\Delta}+\Phi\right), \quad n=1,2, \cdots, N-1,
\end{aligned}\right.
$$

其中, $\theta_{\Sigma}=\theta_{x}+\theta_{y}, \theta_{\Delta}=\theta_{x}-\theta_{y}$. 当 $\Phi=0$ 时,

$$
\left\{\begin{array}{l}
\omega_{2 m+1,2 n+1}=\omega_{\mathrm{ref}}, \\
\omega_{2 m, 2 n}=\omega_{\mathrm{ref}}, \\
\omega_{1,1}=\omega_{\mathrm{ref}}-\Delta \omega_{m} \sin \left(\theta_{x}+\theta_{y}\right), \\
\omega_{2 M, 2 N}=\omega_{\mathrm{ref}}+\Delta \omega_{m} \sin \left(\theta_{x}+\theta_{y}\right), \\
\omega_{2 m-1,1}=\omega_{\mathrm{ref}}-2 \Delta \omega_{m} \sin \theta_{y} \cos \theta_{x}, m=2,3, \cdots, M, \\
\omega_{2 m, 2 N}=\omega_{\mathrm{ref}}+2 \Delta \omega_{m} \sin \theta_{y} \cos \theta_{x}, m=1,2, \cdots, M-1, \\
\omega_{1,2 n-1}=\omega_{\mathrm{ref}}-2 \Delta \omega_{m} \sin \theta_{x} \cos \theta_{y}, n=2,3, \cdots, N, \\
\omega_{2 M, 2 n}=\omega_{\mathrm{ref}}+2 \Delta \omega_{m} \sin \theta_{x} \cos \theta_{y}, n=1,2, \cdots, N-1 .
\end{array}\right.
$$

当所有振荡器的自由振荡频率等于 $\omega_{\mathrm{ref}}$ 时, 只需要对阵列边界上的振荡器的振荡频率进行调 整, 即可得到均匀的平面相位分布.

图 5 为 $\omega_{\mathrm{ref}}=2.45 \mathrm{GHz}$, 锁相带宽为 $160 \mathrm{MHz}, \Phi=0,\left(\theta_{x}, \theta_{y}\right)=\left(80^{\circ}, 5^{\circ}\right)$ 时, $\omega_{m n}$ 按照 (13) 式取值时 $4 \times 4$ 阵列的稳定过程的仿真结果. 图 6 为 $\left(\theta_{x}, \theta_{y}\right)=\left(5^{\circ}, 80^{\circ}\right)$ 时的仿真结果. 其中 图 5(a), 6(a)为阵列相位分布随时间变化过程, 图 5(b), 6(b)为阵列稳定后的相位分布. 


\section{3 相位控制误差分析}

工程实现时, 由于频率控制参量的随机分布, 实际形成的相位分布与期望的相位分布存 在着误差. 在这里, 仅对相邻阵元之间的相位差较小并且 $\Phi=0$ 时的相位控制误差进行分析.

假定所有的 $\omega_{m n}$ 为相互独立, 均值为 $\mu_{0}$, 方差为 $\sigma_{0}^{2}$ 的 Gauss 分布随机变量. 不失一般性, 设 $\mu_{0}=0$ (若 $\mu_{0} \neq 0$, 可以通过(14)式的变换使得 $\left.\mu_{0}=0\right)$,

$$
\theta_{m n}^{\prime}=\theta_{m n}-\mu_{0} t, \quad \omega_{m n}^{\prime}=\omega_{m n}-\mu_{0} .
$$

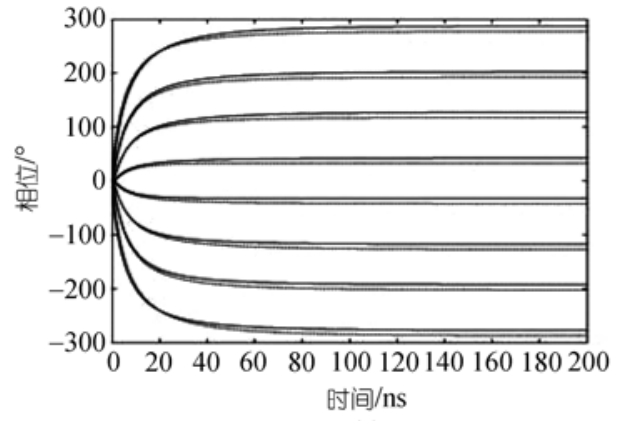

(a)

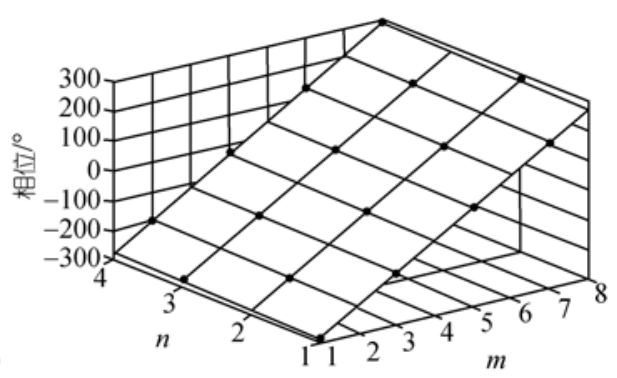

(b)

图 $5\left(\theta_{x}, \theta_{y}\right)=\left(80^{\circ}, 5^{\circ}\right)$ 时 $4 \times 4$ 阵列的相位控制仿真结果

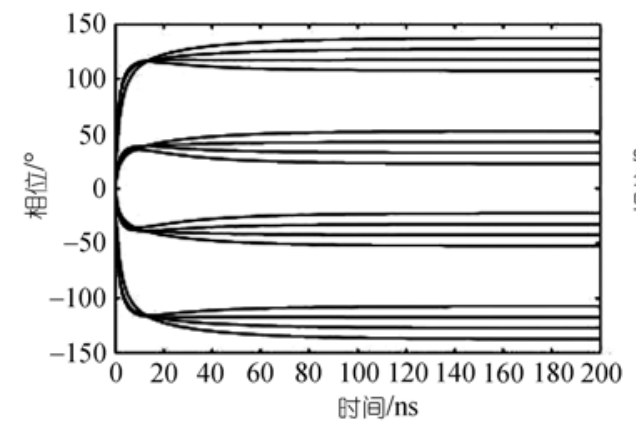

(a)

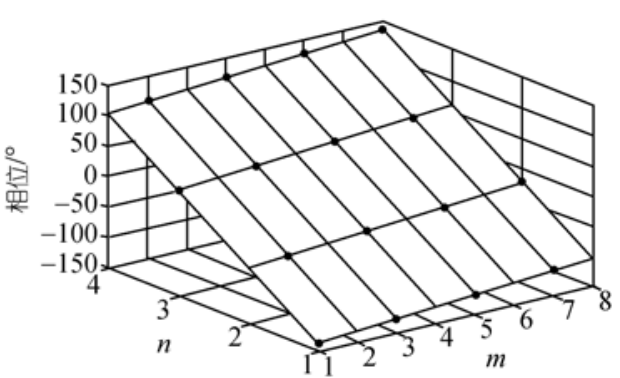

(b)

图 $6\left(\theta_{x}, \theta_{y}\right)=\left(5^{\circ}, 80^{\circ}\right)$ 时 $4 \times 4$ 阵列的相位控制仿真结果

选择 $\theta_{11}$ 为相位参考单元, 即 $\theta_{\text {ref }}=\theta_{11}$. 令 $\Delta \theta_{m n}=\theta_{m n}-\theta_{\text {ref }}=\theta_{m n}-\theta_{11}, \Delta \omega_{m n}=\omega_{m n}-\omega_{11}$. 根据假设, $\sin \left(\theta_{i j}-\theta_{m n}\right) \approx \theta_{i j}-\theta_{m n}, \theta_{i j}$ 为与 $\theta_{m n}$ 相邻的振荡器的相位. 将(7)式中的每个微分方 程减去描述 $\theta_{11}$ 的微分方程得到

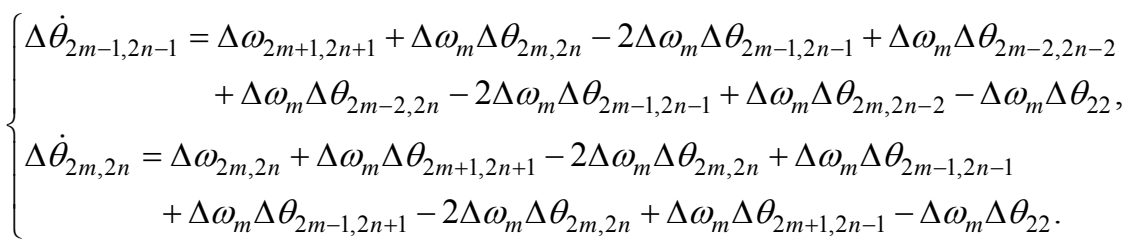


令

$$
\begin{aligned}
& \Delta \theta_{1}=\left(\begin{array}{llll}
\Delta \theta_{3,1} & \Delta \theta_{5,1} & \cdots & \Delta \theta_{2 M-1,1}
\end{array}\right), \Delta \theta_{2}=\left(\begin{array}{lllll}
\Delta \theta_{2,2} & \Delta \theta_{4,2} & \cdots & \Delta \theta_{2 M, 2}
\end{array}\right), \cdots, \\
& \Delta \theta_{2 N-1}=\left(\begin{array}{lllllll}
\Delta \theta_{1,2 N-1} & \Delta \theta_{3,2 N-1} & \cdots & \Delta \theta_{2 M-1,2 N-1}
\end{array}\right), \Delta \theta_{2 N}=\left(\begin{array}{lllll}
\Delta \theta_{2,2 N} & \Delta \theta_{4,2 N} & \cdots & \Delta \theta_{2 M, 2 N}
\end{array}\right) . \\
& \Delta \omega_{1}=\left(\begin{array}{lllllll}
\Delta \omega_{3,1} & \Delta \omega_{5,1} & \cdots & \Delta \omega_{2 M-1,1}
\end{array}\right), \Delta \omega_{2}=\left(\begin{array}{lllll}
\Delta \omega_{2,2} & \Delta \omega_{4,2} & \cdots & \Delta \omega_{2 M, 2}
\end{array}\right), \cdots, \\
& \Delta \omega_{2 N-1}=\left(\begin{array}{llllll}
\Delta \omega_{1,2 N-1} & \Delta \omega_{3,2 N-1} & \cdots & \Delta \omega_{2 M-1,2 N-1}
\end{array}\right), \Delta \omega_{2 N}=\left(\begin{array}{llll}
\Delta \omega_{2,2 N} & \Delta \omega_{4,2 N} & \cdots & \Delta \omega_{2 M, 2 N}
\end{array}\right) .
\end{aligned}
$$

再令

$$
\Delta \boldsymbol{\theta}=\left(\Delta \theta_{1} \Delta \theta_{2} \cdots \Delta \theta_{2 N-1} \Delta \theta_{2 N}\right)^{\mathrm{T}}, \Delta \boldsymbol{\omega}=\left(\Delta \omega_{1} \Delta \omega_{2} \cdots \Delta \omega_{2 N-1} \Delta \omega_{2 N}\right)^{\mathrm{T}} .
$$

将(15)式写成矩阵形式为

$$
\Delta \dot{\boldsymbol{\theta}}=\Delta \boldsymbol{\omega}+\Delta \omega_{m} \boldsymbol{M} \Delta \boldsymbol{\theta}
$$

其中, $M$ 为常数矩阵.

在稳定状态, 有 $\Delta \dot{\boldsymbol{\theta}}=\mathbf{0}$, 从而有

$$
\Delta \boldsymbol{\theta}=-\frac{1}{\Delta \omega_{m}} \boldsymbol{M}^{-1} \Delta \boldsymbol{\omega}
$$

$\Delta \boldsymbol{\theta}$ 的均值和协方差矩阵分别为

$$
\begin{gathered}
E(\Delta \boldsymbol{\theta})=\mathbf{0}, \\
C(\Delta \boldsymbol{\theta})=\frac{1}{\Delta \omega_{m}^{2}} \boldsymbol{M}^{-1} E\left(\Delta \boldsymbol{\omega} \Delta \boldsymbol{\omega}^{\prime}\right)\left(\boldsymbol{M}^{-1}\right)^{\prime}=-\frac{\sigma_{0}^{2}}{\Delta \omega_{m}{ }^{2}} \boldsymbol{P},
\end{gathered}
$$

其中, $P$ 为常数矩阵, 并且 $\boldsymbol{P}=\boldsymbol{M}^{-1} \boldsymbol{Q}\left(\boldsymbol{M}^{-1}\right)^{\prime}$, 这里

$$
\boldsymbol{Q}=\left(\begin{array}{ccccc}
2 & 1 & \cdots & \cdots & 0 \\
1 & 2 & 1 & \cdots & \vdots \\
\vdots & 1 & 2 & \cdots & \vdots \\
\vdots & \cdots & \cdots & \cdots & 1 \\
0 & \cdots & \cdots & 1 & 2
\end{array}\right) .
$$

从而 $\operatorname{Var}\left(\theta_{11}\right)=0, \operatorname{Var}\left(\theta_{m n}\right),(m, n) \neq(1,1)$ 为 $C(\Delta \boldsymbol{\theta})$ 对角线上的值. 图 7 是用 $\sigma_{0}^{2} / \Delta \omega_{m}^{2}$ 归一化 的 $4 \times 4$ 阵列的相位控制误差.

由图 7 可知, 距离参考阵元越远, 相位控制误差越大. 当阵列规模增大时, 相位控制误差 将会增大. 由(19)式可知, 相位控制误差与 $\Delta \omega_{m}^{2}$ 成反比, 增大锁相带宽可以减小相位控制误差. 菱形结构的二维耦合振荡器阵列应用于有源天线阵时, 相位误差可以引起副瓣电压抬高, 波 束展宽以及波束形状的不对称，因此必须在相位误差和锁相带宽之间进行权衡.

\section{4 稳定性分析}

为了使(11)式的均匀平面相位分布成为(7)式的稳态解, 还需要对(7)式进行稳定性分析. 令 


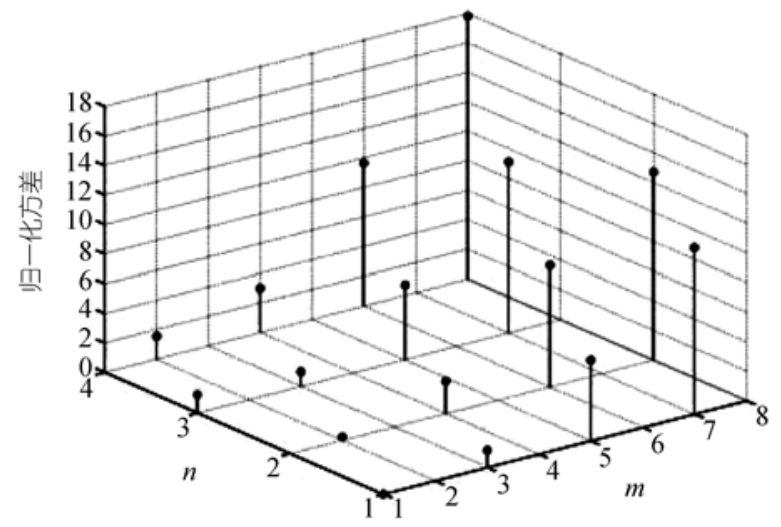

图 $74 \times 4$ 阵列的归一化相位控制误差

$$
\left\{\begin{array}{l}
\theta_{2 m-1,2 n-1}=\theta_{\text {ref }}+(2 m-2) \theta_{x}+(2 n-2) \theta_{y}+\omega_{\text {ref }} t+\eta_{2 m-1,2 n-1}, \\
\theta_{2 m, 2 n}=\theta_{\text {ref }}+(2 m-1) \theta_{x}+(2 n-1) \theta_{y}+\omega_{\text {ref }} t+\eta_{2 m, 2 n},
\end{array}\right.
$$

其中, $\eta_{m n}$ 为微小扰动. 将(20)式代入(7)式, 并取一阶近似, 可以得到线性化稳定性方程为

$$
\left\{\begin{array}{c}
\dot{\eta}_{2 m-1,2 n-1}=\Delta \omega_{m} a_{\Sigma} \eta_{2 m, 2 n}+\Delta \omega_{m} b_{\Sigma} \eta_{2 m-1,2 n-1}+\Delta \omega_{m} c_{\Sigma} \eta_{2 m-2,2 n-2} \\
+\Delta \omega_{m} a_{\Delta} \eta_{2 m, 2 n-2}+\Delta \omega_{m} b_{\Delta} \eta_{2 m-1,2 n-1}+\Delta \omega_{m} c_{\Delta} \eta_{2 m-2,2 n}, \\
\dot{\eta}_{2 m, 2 n}=\Delta \omega_{m} a_{\Sigma} \eta_{2 m+1,2 n+1}+\Delta \omega_{m} b_{\Sigma} \eta_{2 m, 2 n}+\Delta \omega_{m} c_{\Sigma} \eta_{2 m-1,2 n-1} \\
+\Delta \omega_{m} a_{\Delta} \eta_{2 m+1,2 n-1}+\Delta \omega_{m} b_{\Delta} \eta_{2 m, 2 n}+\Delta \omega_{m} c_{\Delta} \eta_{2 m-1,2 n+1} .
\end{array}\right.
$$

其中,

$$
\left\{\begin{array} { l } 
{ a _ { \Sigma } = \operatorname { c o s } ( \theta _ { \Sigma } + \Phi ) , } \\
{ c _ { \Sigma } = \operatorname { c o s } ( \theta _ { \Sigma } - \Phi ) , } \\
{ b _ { \Sigma } = - ( a _ { \Sigma } + c _ { \Sigma } ) , }
\end{array} \quad \left\{\begin{array}{l}
a_{\Delta}=\cos \left(\theta_{\Delta}+\Phi\right) \\
c_{\Delta}=\cos \left(\theta_{\Delta}-\Phi\right) \\
b_{\Delta}=-\left(a_{\Delta}+c_{\Delta}\right)
\end{array}\right.\right.
$$

令

$$
\begin{aligned}
& \eta_{1}=\left(\begin{array}{llll}
\eta_{1,1} & \eta_{3,1} & \ldots & \eta_{2 M-1,1}
\end{array}\right), \eta_{2}=\left(\begin{array}{lllll}
\eta_{2,2} & \eta_{4,2} & \ldots & \eta_{2 M, 2}
\end{array}\right), \cdots, \\
& \eta_{2 N-1}=\left(\begin{array}{llllll}
\eta_{1,2 N-1} & \eta_{3,2 N-1} & \ldots & \eta_{2 M-1,2 N-1}
\end{array}\right), \eta_{2 N}=\left(\begin{array}{llll}
\eta_{2,2 N} & \eta_{4,2 N} & \ldots & \eta_{2 M, 2 N}
\end{array}\right),
\end{aligned}
$$

再令 $\boldsymbol{\eta}=\left(\eta_{1} \eta_{2} \cdots \eta_{2 N-1} \eta_{2 N}\right)^{\mathrm{T}}$, 将(21)式写成矩阵形式, 得到

$$
\dot{\eta}=\Delta \omega_{m} J \boldsymbol{\eta}
$$

其中,

$$
\boldsymbol{J}=\left(\begin{array}{ccccc}
J_{b}(1) & J_{a}(1) & & & \\
J_{c}(2) & J_{b}(2) & J_{a}(2) & & \\
& J_{c}(i) & J_{b}(i) & J_{a}(i) & \\
& & & \ldots & J_{a}(2 N-1) \\
& & & J_{c}(2 N) & J_{b}(2 N)
\end{array}\right)_{2 M N \times 2 M N} .
$$


这里,

$$
\begin{aligned}
& J_{b}(1)=\left(\begin{array}{llll}
-a_{\Sigma} & & & \\
& -a_{\Sigma}-a_{\Delta} & & \\
& & \ldots & \\
& & & -a_{\Sigma}-a_{\Delta}
\end{array}\right), \quad J_{b}(2 j)=\left(\begin{array}{llll}
b_{\Sigma}+b_{\Delta} & & & \\
& \ldots & & \\
& & b_{\Sigma}+b_{\Delta} & \\
& & & -c_{\Sigma}-a_{\Delta}
\end{array}\right), \\
& J_{b}(2 j+1)=\left(\begin{array}{cccc}
-a_{\Sigma}-c_{\Delta} & & & \\
& b_{\Sigma}+b_{\Delta} & & \\
& & \cdots & \\
& & & b_{\Sigma}+b_{\Delta}
\end{array}\right), J_{b}(2 N)=\left(\begin{array}{llll}
-c_{\Sigma}-c_{\Delta} & & & \\
& \cdots & & \\
& -c_{\Sigma}-c_{\Delta} & \\
& & & -c_{\Sigma}
\end{array}\right) \text {, } \\
& J_{a}(2 j-1)=\left(\begin{array}{cccc}
a_{\Sigma} & & & 0 \\
a_{\Delta} & a_{\Sigma} & & \\
& a_{\Delta} & \ldots & \\
0 & & a_{\Delta} & a_{\Sigma}
\end{array}\right)_{M \times M}, J_{a}(2 j)=\left(\begin{array}{cccc}
a_{\Delta} & a_{\Sigma} & & 0 \\
& a_{\Delta} & a_{\Sigma} & \\
& & \ldots & a_{\Sigma} \\
0 & & & a_{\Delta}
\end{array}\right)_{M \times M}, \\
& J_{c}(2 j-1)=\left(\begin{array}{cccc}
c_{\Delta} & \cdots & & 0 \\
c_{\Sigma} & c_{\Delta} & \cdots & \\
& c_{\Sigma} & \cdots & \\
0 & \cdots & c_{\Sigma} & c_{\Delta}
\end{array}\right)_{M \times M}, J_{c}(2 j)=\left(\begin{array}{cccc}
c_{\Sigma} & c_{\Delta} & \cdots & 0 \\
& c_{\Sigma} & c_{\Delta} & \\
& \cdots & & c_{\Delta} \\
0 & \cdots & & c_{\Sigma}
\end{array}\right)_{M \times M} \text {. }
\end{aligned}
$$

从而, 稳定性矩阵 $J$ 为 $2 M N \times 2 M N$ 阶方阵, 并且是对角占优的稀疏矩阵. 记 $J=\left(p_{i j}\right)_{2 M N \times 2 M N}$, 该矩阵每一行的所有元素之和等于 0 . 根据 Gerschgorin 定理, $J$ 的特征值均在复平面上的 $2 M N$ 个 Gehr 圆 $\left|\lambda-p_{i i}\right| \leqslant R_{i}(i=1,2, \cdots, 2 M N)$ 的并集内, 这里

$$
R_{i}=\sum_{j=1, j \neq i}^{M N}\left|p_{i j}\right| .
$$

当菱形耦合振荡器阵列的耦合相位 $\Phi=0$ 时, 并且

$$
\left|\theta_{x}+\theta_{y}\right| \leqslant \frac{\pi}{2} \text { 且 }\left|\theta_{x}-\theta_{y}\right| \leqslant \frac{\pi}{2}
$$

时, 有

$$
\begin{gathered}
p_{i i} \leqslant 0, \quad p_{i j} \geqslant 0(j \neq i), \\
p_{i i}+R_{i}=p_{i i}+\sum_{j=1, j \neq i}^{M N}\left|p_{i j}\right|=\sum_{j=1 i}^{M N}\left|p_{i j}\right|=0 .
\end{gathered}
$$

从而 $2 M N$ 个 Gehr 圆位于左半平面内, 或者退化为原点. 等于 0 的特征值表示沿解的轨道方向 的扰动, 不影响方程的稳定性; 除此之外, $J$ 的特征值均位于左半平面内, 因此系统稳定.

\section{3 菱形结构阵列与矩形栅格结构阵列性能比较}

\section{1 波束扫描范围}

考虑图 8(a)所示的阵元按照菱形结构排列的有源天线阵, 该阵列可以看成由两个阵元间 
距为 $d_{r x}$ 和 $d_{r y}$ 的矩形天线阵组成, 两个阵列在 $x$ 和 $y$ 方向上位置分别相差 $0.5 d_{r x}, 0.5 d_{r y}{ }^{[11]}, \theta_{r x}$ 和 $\theta_{r y}$ 分别为 $x$ 和 $y$ 方向上相邻阵元的相位差. 图 8(b)为阵元按照矩形栅格结构排列的有源天线阵, $d_{g x}$ 和 $d_{g y}$ 分别为 $x$ 和 $y$ 方向上相邻阵元的间距, $\theta_{g x}$ 和 $\theta_{g y}$ 分别为分别为行和列方向上相邻阵元的相位 差. $x$ 为水平方向, $y$ 为俯仰方向.

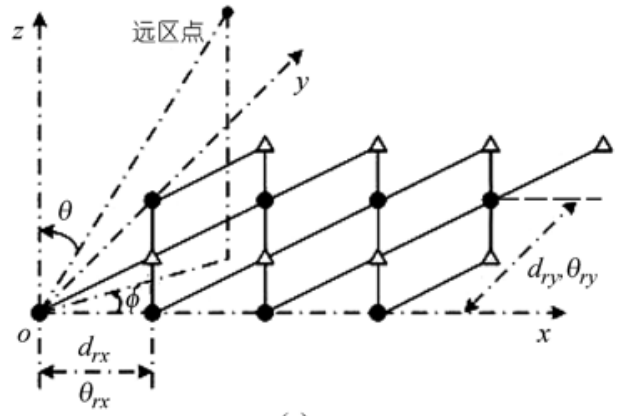

(a)

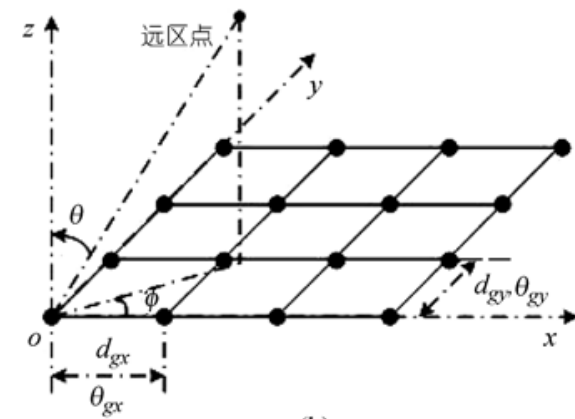

(b)

图 8 阵元按菱形结构和矩形栅格结构排列的天线阵几何关系

菱形结构阵列的方向图为

$$
F(\theta, \phi)=F_{s}(\theta, \phi) F_{0}(\theta, \phi),
$$

其中, $F_{0}(\theta, \phi)=\sum_{m=1}^{M} \sum_{n=1}^{N} \exp \left(\mathrm{j} k_{0}\left(d_{r x} u+d_{r y} v\right)\right), \quad F_{s}(\theta, \phi)=1+\exp \left(\mathrm{j} k_{0}\left(0.5 d_{r x} u+0.5 d_{r y} v\right)\right) . \quad k_{0}=$ $2 \pi / \lambda, \quad u=\sin \theta \cos \phi-\sin \theta_{B} \cos \phi_{B}, \quad v=\sin \theta \sin \phi-\sin \theta_{B} \sin \phi_{B} . \quad \theta$ 和 $\phi$ 分别表示俯仰角和方位 角, $\left(\theta_{B}, \phi_{B}\right)$ 为波束指向.

根据(11)式的菱形结构耦合振荡器阵列的相位分布，有

$$
\begin{aligned}
F(\theta, \phi)= & {\left[1+\exp \left(\mathrm{j}\left(0.5 k_{0} d_{r x} \sin \theta \cos \phi-0.5 \theta_{r x}+0.5 k_{0} d_{r y} \sin \theta \sin \phi-0.5 \theta_{r y}\right)\right)\right] } \\
& \times \sum_{m=1}^{M} \sum_{n=1}^{N} \exp \left(\mathrm{j}\left(k_{0} d_{r x} \sin \theta \cos \phi-\theta_{r x}+k_{0} d_{r y} \sin \theta \sin \phi-2 \theta_{r y}\right)\right) .
\end{aligned}
$$

波束指向与相位差的关系为

$$
\left\{\begin{array}{l}
\theta_{r x}=k_{0} d_{r x} \sin \theta_{B} \cos \phi_{B} \\
\theta_{r y}=k_{0} d_{r y} \sin \theta_{B} \sin \phi_{B}
\end{array}\right.
$$

对图 8(b)所示的对于矩形栅格结构阵列, 其波束指向与相位差的关系为

$$
\left\{\begin{array}{l}
\theta_{g x}=k_{0} d_{g x} \sin \theta_{B} \cos \phi_{B}, \\
\theta_{g y}=k_{0} d_{g y} \sin \theta_{B} \sin \phi_{B} .
\end{array}\right.
$$

根据(11)式及(23)式的稳定性条件, 有 $\theta_{r x}=2 \theta_{x}, \theta_{r y}=2 \theta_{y}$, 并且

$$
\left|\theta_{r x}+\theta_{r y}\right| \leqslant \pi,\left|\theta_{r x}-\theta_{r y}\right| \leqslant \pi .
$$

菱形结构和矩形栅格结构的阵元间距相同时, 即 $d_{r x}=d_{g x}, d_{r y}=d_{g y}$ 时, 结合(6)式, 由于 菱形结构所能形成的最大相位差比矩形栅格结构的大, 因而具有比矩形栅格结构更宽的波束 
扫描范围.

\section{2 栅瓣}

对图 8(a)所示的菱形结构阵列, 当波束扫描至 $\left(\theta_{B}, \phi_{B}\right)$ 时, 出现栅瓣的条件为

$$
\begin{cases}0.5 k_{0} d_{r x}\left(\sin \theta \cos \phi-\sin \theta_{B} \cos \phi_{B}\right)=p \pi, & p= \pm 1, \pm 2, \cdots, \\ 0.5 k_{0} d_{r y}\left(\sin \theta \sin \phi-\sin \theta_{B} \sin \phi_{B}\right)=q \pi, & q= \pm 1, \pm 2, \cdots .\end{cases}
$$

且 $p+q$ 为偶数.

因为如果 $p+q$ 为奇数, 则 (24) 式中 $F_{s}(\theta, \phi)=0$, 从而 $F(\theta, \phi)=0$, 不会出现栅瓣. $p=q=0$ 表示天线主瓣的情况. (29)式又可以写成

$$
\begin{cases}\sin \theta \cos \phi=\sin \theta_{B} \cos \phi_{B}+p \lambda / d_{r x}, & p= \pm 1, \pm 2, \cdots, \\ \sin \theta \sin \phi=\sin \theta_{B} \sin \phi_{B}+q \lambda / d_{r y}, & q= \pm 1, \pm 2, \cdots .\end{cases}
$$

对图 8(b)所示的对于矩形栅格结构阵列, 出现栅瓣的条件为

$$
\begin{cases}\sin \theta \cos \phi=\sin \theta_{B} \cos \phi_{B}+p \lambda / d_{g x}, & p= \pm 1, \pm 2, \cdots, \\ \sin \theta \sin \phi=\sin \theta_{B} \sin \phi_{B}+q \lambda / d_{g y}, & q= \pm 1, \pm 2, \cdots,\end{cases}
$$

但没有 $p+q$ 为偶数的限制. 从而与矩形栅格结构阵列相比, 菱形结构的阵列的无栅瓣波束扫 描范围较宽.

\section{3 需要调整的阵元数}

对菱形结构和矩形栅格结构而言, 当所有振荡器的自由振荡频率都相等时, 只需要对阵 列边界上的振荡器的振荡频率进行调整, 即可得到均匀的平面相位分布.

对于 $M \times 2 N$ 的阵列而言, 要形成均匀的平面相位分布, 根据(11)式, 菱形结构需要调整的 阵元数目为 $2 M+2(N-1)$ 个, 而矩形栅格结构需要调整的阵元数目为 $2 M+4(N-1)$ 个. 对于菱形 结构, 所需要的耦合网络的个数为 $(2 N-1)(2 M-1)$ 个, 而矩形栅格结构需要的耦合网络为 $2 N(M-1)+M(2 N-1)$ 个. 同样规模的阵列, 菱形结构比矩形栅格结构需要调整的阵元数目减少 了 $2(N-1)$ 个, 所需要的耦合网络数目减少了 $M-1$ 个.

\section{4 结束语}

小型化、低成本相控阵天线是相控阵雷达技术发展的重要方向. 基于耦合振荡器阵列的有 源相控阵天线由于不采用移相器, 利于集成, 因而是相控阵天线实现低成本和小型化的有效 途径之一. 本文提出了一种菱形结构的二维耦合振荡器阵列, 在保留耦合振荡器阵列基本特 点的同时, 不需要对阵列的输出进行倍频, 水平和俯仰方向上相邻阵元的相位差范围就可以 达到 $\left[-180^{\circ}, 180^{\circ}\right]$, 从而具有比矩形栅格结构更宽的波束扫描范围. 文中推导了其相位控制方 法并对稳定性进行了分析, 同时, 对相位控制误差进行了分析. 从分析结果看, 耦合振荡器阵 列的相位误差具有积累效应, 距离参考阵元越远, 相位误差越大. 在大规模相控阵天线中采用 耦合振荡器阵列时, 难以实现较低的副瓣. 同时, 由于阵列的相位控制具有一定的惯性, 阵列 规模越大, 阵列的稳定过程越长, 不利于波束快速扫描. 这也限制了其在大规模相控阵天线中 
的应用. 采用幅度加权是降低副瓣电压的一种可行方案, 但会增加系统的复杂性和成本. 当波 束快速扫描时，可以通过对频率控制参量进行修正的方法实现无惯性的波束扫描. 如何对耦 合振荡器阵列的相位误差进行控制, 是需要进一步研究的课题.

\section{参考文献}

1 Liao P, York R A. A new phase shifterless beam-scanning technique using arrays of coupled oscillators. IEEE Trans Microw Theory Tech, 1993, 41: 1810-1815 [DOI]

2 York R A. Nonlinear analysis of phase relationships in quasi-optical oscillator arrays. IEEE Trans Microw Theory Tech, 1993, 41: 1799-1809 [DO]

3 Adler R. A study of locking phenomena in oscillators. In: Proc IEEE, vol 61(10). New York: IEEE Press, 1973. $1380-1385$

4 Pogorzelski R J, Scaramastra R P, Huang J, et al. A seven element s-band coupled oscillator controlled agile beam phased array. IEEE Trans Microw Theory Tech, 2000,48(8): 1375-1384 [DOI]

5 Heath T, Wiesenfeld K, York R A. Manipulated synchronization: beam steering in phased arrays. Int J Bifurcation Chaos, 2000,10: 2619-2627

6 Pogorzelski R J. On the dynamics of two-dimensional array beam scanning via perimeter detuning of coupled oscillator arrays. IEEE Trans Antennas Propag, 2001, 49(2): 234-242 [DOI]

7 Heath T, Kerr R R, Hopkins G D. Two-dimensional, nonlinear oscillator array antenna. In: Proceedings of IEEE Aerospace Conference. Big Sky, MT: IEEE Press, 2005. 1104-1115

8 Pogorzelski R J. A two-dimensional coupled oscillator array. IEEE Microw Guid Wave Lett., 2000,10(11): 478480 [DOI]

9 Pogorzelski R J. A frequency tripled two-dimensional coupled oscillator array. IEEE Trans Antennas Propag, 2004, 52(3): 907-910 [DOI]

10 Pogorzelski R J. Phased arrays based on oscillators coupled on triangular and hexagonal lattices. IEEE Trans Antennas Propag, 2004, 52(3): 790-800 [DOI]

11 张广义. 相控阵雷达系统. 北京: 国防工业出版社, 1994. 57-59 